Caroline Fatoux Université Laval

Martine Mottet

Université Laval

Soufiane Rouissi

Université Bordeaux Montaigne

\section{État des connaissances sur la demande d'aide : quel apport pour la formation universitaire en ligne?}

Help seeking in higher education online courses: a state of knowledge review

\section{2 ésumé}

Formuler une demande d'aide de manière autorégulée peut avoir un impact positif sur l'apprentissage et la réussite. Cependant, peu d'études ont été menées sur ce sujet dans un contexte d'enseignement supérieur à distance, qui pose pourtant plusieurs défis aux étudiants. Nous faisons part dans le présent article de notre recension d'écrits sur les demandes d'aides exprimées en classe et en ligne, ainsi que sur la communication par courriel, puis nous suggérons des pistes de recherche.

\section{Mots-clés}

Demande d'aide, autorégulation, courriel, forum, enseignement universitaire, formation à distance, apprentissage en ligne, réussite académique.

Abstract

Self-regulated help seeking may have a positive impact on learning and academic achievement.

However, few studies have been conducted on this subject in a higher distance education

context although it usually raises several challenges to learners. We share in this article our literature review focusing on help seeking in classroom and online environments, as well as on email communication, then we conclude by suggesting research avenues

Keywords

Help-seeking behavior, self-regulation, email, forum, higher education, distance education, e-learning, academic achievement

\section{Problématique}

Les universités offrent de plus en plus de cours en ligne, participant ainsi à l'essor actuel de l'offre de formation à distance (Saucier, 2013). Comme le taux d'abandon y est cependant généralement supérieur à celui de la formation en présentiel (Park et Choi, 2009; Poellhuber, 2007), la persévérance et la réussite des étudiants font l'objet de nombreuses recherches. Or, plusieurs études ont montré que les étudiants qui réussissent bien sont capables de mobiliser des stratégies d'apprentissage cognitives, métacognitives, affectives et de gestion des ressources (Pintrich, Smith, Garcia et McKeachie, 1993). Parmi les stratégies métacognitives, l'autorégulation-soit la capacité de s'adapter aux situations d'apprentissage rencontrées en contrôlant, observant et évaluant ses activités cognitives, sa motivation et son comportement (Pintrich, 1995, 2000) - influence favorablement l'apprentissage. Ainsi, l'étudiant qui fait preuve d'autorégulation demande de l'aide lorsqu'il rencontre une difficulté, de manière à obtenir une réponse qui lui permette de la surmonter, ce qui favorise son apprentissage et sa réussite académique (Arbreton, 1998; Karabenick, 1998; Karabenick et Newman, 2006; Roll, Aleven, McLaren et Koedinger, 2011; Ryan, Pintrich et Midgley, 2001; Zimmerman et Schunk, 2001).

Or, la formation en ligne exige plus d'autonomie et d'autorégulation qu'en classe (Cosnefroy, 2012; Jézégou, 2010). Les étudiants qui suivent un cours en ligne ont par conséquent particulièrement besoin d'être soutenus et guidés (Kitsantas et Chow, 2007; Koc et Liu, 2016) et une meilleure compréhension de leur comportement de demandeur d'aide pourrait permettre aux professeurs de mieux les accompagner dans leur apprentissage (Koc et Liu, 2016). En effet, la demande d'aide formulée dans un cours en ligne est différente par plusieurs aspects de celle formulée en classe (Koc et Liu, 2016); des facteurs tels que la technologie ou le système d'environnement 
d'apprentissage pouvant en effet avoir une influence sur celle-ci (Koc et Liu, 2016). Notre expérience de la formation à distance en contexte universitaire nous montre aussi que les étudiants ne savent pas toujours adresser adéquatement leurs demandes d'aide à leur enseignant. Ainsi, un apprenant qui envoie un courriel avec pour seul contenu les mots « Pouvez-vous maider? » révèle sa difficulté à cerner son besoin et à s'autoréguler. Pourtant, bien que les besoins d'aide augmentent avec l'essor des cours en ligne et semblent spécifiques à ce contexte d'apprentissage, la demande d'aide demeure peu étudiée en formation à distance universitaire (Puustinen, 2012).

Dans cet article, nous présentons l'état des connaissances actuelles sur les demandes d'aide et les courriels adressés par les apprenants de différents niveaux scolaires à leur enseignant dans le cadre de cours en classe et à distance. Nous nous penchons sur diverses populations étudiantes, soit les apprenants du primaire, du secondaire et de l'université. En effet, bien que conscients des possibles différences entre ces publics, nous pensons que les études portant sur les demandes d'aide des élèves du primaire et du secondaire, largement plus nombreuses que celles portant sur les étudiants universitaires, peuvent servir de base de réflexion pour mieux comprendre la demande d'aide à l'université. En outre, les études portant sur la demande d'aide à distance étant également peu nombreuses, nous avons élargi notre recension des écrits au contexte de la classe.

\section{Méthodologie}

Nous avons mené cette recension d'écrits en nous fondant sur la méthode Evidence for Policy and Practice Information (EPPI) (Gough, Oliver et Thomas, 2013), qui permet d'effectuer une revue de littérature systématique. Tout d'abord, nous avons identifié les thèmes de recherche (la demande d'aide et la formation en ligne à l'université) et ciblé les bases de données Education Source et ERIC. Puis, pour délimiter notre corpus, nous avons choisi des mots-clés en français et en anglais et les avons combinés grâce à des opérateurs booléens. Nous avons également choisi de retenir au sujet de la demande d'aide les articles publiés depuis l'an 2000, une période assez longue qui s'explique par la rareté des études sur ce sujet. Nous avons ensuite réduit le corpus en éliminant les doublons et en excluant les articles qui ne présentaient pas de données empiriques.

En nous fondant sur cette recension d'écrits, nous mènerons dans un deuxième temps une recherche exploratoire, qui visera à décrire les demandes d'aide d'étudiants universitaires inscrits à un cours en ligne et, par la suite, à aider ceux-ci à mieux formuler leurs demandes, et ce, dans une perspective d'appui à la réussite.

\section{Stratégies d'apprentissage mobilisées dans les demandes d'aide}

C'est avec les travaux fondateurs de Nelson-Le Gall (1981) sur les enfants que la demande d'aide est perçue non plus comme une manifestation de la dépendance de l'apprenant à l'égard de son enseignant, mais bien de manière positive comme une stratégie à laquelle il recourt afin de surmonter une difficulté et d'apprendre. Depuis, plusieurs chercheurs ont montré que ce processus nécessite de mettre en œuvre des stratégies métacognitives, affectives et de gestion des ressources. 
Tout d'abord, demander de l'aide dans un contexte d'apprentissage fait en effet appel à des stratégies métacognitives, et plus précisément à la capacité d'autoréguler son apprentissage (Aleven, Stahl, Schworm, Fischer et Wallace, 2003; Godbole-Chaudhuri, Winters, Azevedo et Hofman, 2006; Puustinen, 2013; Ryan et Pintrich, 1997; Ryan et al., 2001). L'autorégulation correspond au fait de s'auto-observer (Cosnefroy, 2010) pour s'adapter à la situation d'apprentissage (Pintrich, 1995, 2000). Elle s'acquiert avec l'âge et doit être renforcée régulièrement (Tardif, 1997; Thouraya, 2007). Comme nous le verrons plus tard, létudiant fait appel à sa capacité de s'autoréguler tout au long du processus de demande d'aide.

Ensuite, parmi les stratégies affectives favorisant la réussite académique, la motivation est à prendre en compte lorsqu'on s'intéresse à la demande d'aide. Pour étudier cette dernière, les auteurs se sont appuyés sur divers concepts et théories, comme la théorie des buts d'accomplissement (Dweck et Elliott, 1983; Nicholls, 1984), le concept de l'attribution causale (Weiner, 1979) ou encore le sentiment d'efficacité personnelle (Bandura, 1986). Sur cette base, ils ont décrit les caractéristiques des demandeurs d'aide. D'abord,les auteurs s'étant fondés sur la théorie des buts d'accomplissement ont montré que les étudiants qui demandent de l'aide se concentrent plus sur la maîtrise de la matière ou de la tâche (Roussel, Elliot et Feltman, 2011; Ryan et Pintrich, 1997; Ryan et al., 2001) que sur leur performance (c'est-à-dire leurs résultats par rapport à autrui et le jugement que les autres portent sur leurs habiletés). Ils perçoivent également moins le fait de demander de l'aide comme une menace à leur estime de soi, et davantage comme étant bénéfique à leur apprentissage (Ryan et Pintrich, 1997). Puis, selon Ames (1983), qui s'est basé sur le concept de l'attribution causale de Weiner (1979), il existe un lien entre l'explication que donne l'étudiant de sa performance (réussite ou échec à un examen) et son comportement face à la demande d'aide. En effet, selon qu'il attribue ses réussites ou ses échecs à des facteurs internes, comme son habileté ou ses efforts, ou à des facteurs externes et incontrôlables, comme le niveau de difficulté de la tâche ou la chance, il peut percevoir que demander de l'aide augmentera ses chances de succès ou non. Par exemple, un apprenant qui attribue son échec à un manque d'effort de sa part peut être amené à mobiliser plusieurs stratégies, notamment celle de recourir à l'aide d'autrui. Enfin, d'autres auteurs ont étudié la demande d'aide en s'appuyant sur le concept du sentiment d'efficacité personnelle (Pintrich et Schunk, 1996; Ryan, Gheen et Midgley, 1998; Williams et Takaku, 2011). Ils ont montré que les apprenants ayant un sentiment d'efficacité personnelle élevé interprètent moins leur besoin d'aide comme une marque d'incompétence et ont davantage tendance à recourir à de l'aide (Pintrich et Schunk, 1996; Williams et Takaku, 2011).

Enfin, des stratégies de gestion des ressources humaines sont aussi mobilisées lors de la demande d'aide (Pintrich et al., 1993). En effet, l'apprenant doit notamment posséder l'habileté à communiquer son besoin pour que la réponse de l'aidant soit adaptée à sa requête (Kitsantas et Dabbagh, 2009; Roussel et al., 2011). Par conséquent, selon Ryan et Pintrich (1997), les compétences sociales des apprenants, c'est-à-dire leurs compétences à établir des relations et à interagir avec autrui, ont un effet sur la décision de demander de l'aide; les apprenants qui se sentent compétents à communiquer et qui sont à l'aise avec autrui ont moins tendance à se sentir menacés par le regard des autres. 


\section{La demande d'aide}

\section{Types de demandes d'aide}

En plus d'avoir établi l'aspect positif de la demande d'aide, Nelson-Le Gall (1981, 1985) a distingué deux types de demandes d'aide : les demandes d'aide instrumentales, dites aussi autorégulées, et les demandes d'aide substitutives, qui ne sont pas autorégulées. Cette catégorisation a été reprise par plusieurs chercheurs, qu'ils aient mené leur étude en contexte de classe, en enseignement primaire ou secondaire (Puustinen, 2013) ou universitaire (Butler, 2006; Roussel et al., 2011), ou encore en contexte de formation à distance en enseignement secondaire (Puustinen et Bernicot, 2013) ou universitaire (Kitsantas et Dabbagh, 2009).

L'apprenant qui soumet une demande d'aide instrumentale ou autorégulée limite sa demande aux seuls éléments qui lui permettront de résoudre son problème de manière autonome (Kitsantas et Dabbagh, 2009; Puustinen, 2013; Roussel et al., 2011). Les travaux de Puustinen (2013), qui ont porté sur les enfants et les adolescents, ont permis de mettre en évidence deux types de demandes d'aide instrumentales : les demandes d'explication, qui sont formulées de manière générale («Comment fautil citer une statistique? »), et les demandes de clarification, qui sont plus détaillées ("Pour citer une vidéo, est-ce qu'il faut écrire que ça vient de YouTube? »).

La demande substitutive ou non autorégulée est formulée par des apprenants qui souhaitent se débarrasser de la tâche en la faisant faire par autrui (Puustinen, 2013) : («[...] mais je ne sais pas comment citer mon image. Pouvez-vous mécrire la référence?»). Ils souhaitent recevoir la réponse toute faite ou des directives tellement précises que la solution au problème devient évidente (Butler, 2006; Nelson-Le Gall et Glor-Scheib, 1985; Roll et al., 2011).

D'autres types de demandes d'aide non autorégulées ont été relevées lors de recherches récentes qui ont porté sur les enfants dans un contexte de classe et sur les messages envoyés par des adolescents dans un forum en ligne (Puustinen, 2013; Puustinen, Bernicot et Bert-Erboul, 2011). Tout d'abord, la demande de confirmation reflèterait le manque de confiance en soi de l'apprenant, qui souhaite vérifier auprès de l'expert que sa réponse est la bonne ( "J'ai utilisé le masque des diapositives, est-ce que c'est correct?»). Ensuite, la demande d'aide non spécifiée est formulée de manière tellement vague que l'enseignant ne peut savoir de quelle aide a besoin l'apprenant ("Pouvez-vous m'aider? », «SVP répondez-moi le plus vite possible»).

Par ailleurs, le comportement d'évitement de la demande d'aide a aussi été identifié dans plusieurs études (Cheng, Liang et Tsai, 2013; Roll, Baker, Aleven et Koedinger, 2014; Ryan et al., 1998; Ryan et Pintrich, 1997; Ryan et al., 2001). Ce comportement influence négativement l'apprentissage et la performance. Dans un article portant sur la motivation et les attitudes des adolescents au moment de demander de l'aide, Ryan et Pintrich (1997) notent ce comportement, qui peut consister à laisser tomber l'exercice qui pose problème ou encore à donner n'importe quelle réponse à une question.

Enfin, des facteurs internes aux apprenants peuvent être mis en relation avec le type de demande d'aide. Ainsi, quelques chercheurs ont voulu établir des liens entre l'âge et le comportement de demandeur d'aide autorégulé ou non. Par exemple, au primaire, Puustinen (2013) a montré que des élèves de 
9 ans sont plus autorégulés que ceux de 7 ans. Par contre, au secondaire, Marchand et Skinner (2007) ont quant à eux démontré que des élèves évitent de demander de l'aide au début de l'adolescence, ce qui ne correspond pas à un comportement autorégulé. Au niveau universitaire, Karabenick et Knapp (1988b) ont montré dans une étude que la majorité des étudiants évitaient eux aussi de demander de l'aide. Ces résultats semblent indiquer la difficulté à établir avec certitude un lien entre l'âge et le type de demande d'aide. D'autres facteurs, comme le manque de motivation ou la peur d'être jugé, peuvent aussi être mis en relation avec le comportement d'évitement (Deasy, Coughlan, Pironom, Jourdan et Mannix-McNamara, 2016).

Des facteurs externes aux apprenants peuvent également être mis en relation avec le type de demande d'aide. Tout d'abord, l'attitude des professeurs dans leur réponse pourrait amener les apprenants à formuler leurs demandes de manière plus autorégulée. Ainsi, dans une étude portant sur la demande d'aide formulée sur les forums par des adolescents, Puustinen, Bernicot, Volckaert-Legrier et Baker (2015) ont remarqué que certains sont devenus plus autorégulés après leur premier contact avec l'enseignant et émettent l'hypothèse que la qualité de la réponse de celui-ci pourrait contribuer à favoriser l'autorégulation. De plus, l'environnement d'apprentissage en formation à distance étant caractérisé par l'éloignement entre l'apprenant et ses pairs ou ses professeurs, cela peut susciter un sentiment d'isolement (Dunn, Rakes et Rakes, 2014) et faire obstacle à la demande d'aide.

\section{Processus de demande d'aide}

La présentation de la demande n'est cependant qu'une des étapes du processus de demande d'aide élaboré par Nelson-Le Gall (1981, 1985). Depuis, ce modèle a été repris, en tout ou en partie, par plusieurs chercheurs (Godbole-Chaudhuri et al., 2006; Ryan et Pintrich, 1997; Ryan et al., 2001). Parmi eux, Puustinen (2013) l'a enrichi et en propose la version suivante, accompagnée d'indicateurs d'autorégulation :

1. Détection d'une difficulté. L'apprenant prend conscience que les ressources dont il dispose ne sont pas suffisantes pour résoudre son problème.

2. Détermination de la nécessité d'une aide. Une fois conscient de sa difficulté, il s'autoquestionne pour déterminer s'il peut la résoudre seul ou s'il doit demander de l'aide.

3. Choix du type d'aide demandée. En fonction du but qu'il poursuit (résoudre lui-même la tâche ou la faire faire par une autre personne), il choisit ensuite un type de demande d'aide : instrumentale ou substitutive.

4. Demande d'aide à proprement parler. S'il est capable d'autorégulation, il restreint sa demande aux seuls éléments qui lui permettent de résoudre son problème et il restreint aussi le nombre de ses demandes d'aide à ce qui est nécessaire. De plus, sa demande d'aide est explicite et non implicite.

5. Obtention de l'aide. Le demandeur d'aide obtient l'assistance dont il a besoin.

6. Traitement de l'aide obtenue. Enfin, l'apprenant autorégulé traite l'aide obtenue et est capable de la réinvestir dans des tâches similaires. 
Nous venons d'examiner les différents types de demandes d'aide et le processus de demande d'aide qui, dans le cas de la demande d'aide instrumentale, requiert la mobilisation de stratégies autorégulatrices, affectives et sociales. Nous allons à présent nous intéresser aux courriels envoyés par les étudiants dans un contexte d'apprentissage en présentiel et à distance.

\section{Les courriels dans les cours en classe et en ligne}

Puustinen et Rouet (2009) ont identifié trois situations de demande d'aide dans un environnement d'apprentissage informatique. Dans la première, un humain est présent physiquement auprès des apprenants pour répondre à leurs besoins d'aide lorsqu'ils sont devant un ordinateur, par exemple dans une salle informatique. Dans la deuxième, un expert humain répond à la demande d'aide par l'intermédiaire d'un outil de communication informatique, comme le courriel ou le forum de discussion. Dans la troisième, l'humain est remplacé par un dispositif informatique. Les études portant sur cette dernière situation, soit les dispositifs d'aide informatiques dans un environnement d'apprentissage, ont montré que les apprenants les utilisent de manière non efficace ou n'y recourent pas du tout (Aleven et al., 2003; Kitsantas, Dabbagh et Dass, 2013). Nous nous intéressons à la deuxième situation, celle où les demandes d'aide se font à distance par l'intermédiaire d'un outil de communication électronique, soit le courriel.

\section{Nombre de courriels}

Le nombre de courriels envoyés aux enseignants dans les cours universitaires a augmenté considérablement au fil des ans (Bolkan et Holmgren, 2012; Stephens, Houser et Cowan, 2009), et ce phénomène concerne aussi bien les étudiants à distance que les étudiants sur campus, qui préfèrent utiliser le courriel pour adresser ainsi leurs demandes aux enseignants, plutôt que de le faire en face-àface (Karabenick et Knapp, 1988a; Kitsantas et Chow, 2007).

Ce recours fréquent au courriel peut s'expliquer par les nombreux avantages que présente ce moyen de communication. Parmi ceux-ci, il permettrait de favoriser la communication (Hassini, 2006), d'augmenter les interactions et de réduire le stress en n'étant pas dans une situation de face-à-face (White et Le Cornu, 2002), d'envoyer plus d'un message par jour sans craindre de déranger la personne - contrairement au téléphone -, d'envoyer des pièces jointes et d'obtenir une réponse rapide (Sasseville et Morel, 2008). En outre, le courriel serait perçu par les apprenants comme plus accessible que les sources d'aide en face à face (Makara et Karabenick, 2013) et, étant donné son caractère privé, apparaitrait moins menaçant pour les étudiants que de formuler une demande d'aide dans une salle de classe (Karabenick et Knapp, 1988b; Kitsantas et Chow, 2007) ou dans un forum (Makara et Karabenick, 2013). Ainsi, bien que le forum soit de plus en plus utilisé aujourd'hui par les enseignants (Reffay et Lancieri, 2006), une étude de Reeves et Sperling (2015) a montré qu'il est une des sources d'aide les moins désirées par les étudiants, qui préfèrent s'adresser au professeur par courriel ou encore avant ou après la classe. 


\section{Teneur des courriels}

La teneur des messages envoyés par les étudiants est très variée et n'a pas encore fait, à notre connaissance, l'objet d'une classification. Les auteurs évoquent en vrac, par exemple, le contenu du cours, la méthodologie, l'abandon de la formation, les difficultés administratives (Béziat, 2003), ainsi que les demandes de rendez-vous (Bolkan et Holmgren, 2012), de résultats d'examen (BiesenbachLucas, 2007) ou de délai pour remettre un devoir (Woodfield et Economidou-Kogetsidis, 2010).

\section{Rédaction des courriels}

Cependant, le courriel, qui est un moyen de communication mêlant des caractéristiques de l'écrit et de l'oral (Kerbrat-Orecchioni, 2007; Volckaert-Legrier et Bernicot, 2006; Volckaert-Legrier, BertErboul et Bernicot, 2006), ainsi que des marques langagières issues des modes de communication médiatisée par ordinateur (CMO) (Volckaert-Legrier et Bernicot, 2006), peut poser des problèmes de communication pour les étudiants qui demandent de l'aide. En effet, dans un contexte académique en présentiel comme à distance, alors que l'étudiant rédige rapidement son message (Volckaert-Legrier et Bernicot, 2006), le professeur le lit généralement sans se soucier de savoir s'il a été écrit de manière spontanée ou non (Ragan et White, 2001). Ce paradoxe se retrouve également dans l'emploi des formules d'ouverture et de clôture. Sous l'influence de l'oral et des CMO, elles tendent en effet à être plus informelles, plus réduites, voire inexistantes dans les courriels, par rapport à l'écrit traditionnel (Kerbrat-Orecchioni, 2007; Volckaert-Legrier et Bernicot, 2006). Ainsi, dans le corpus quelle a recensé, Kerbrat-Orecchioni (2007) note que la formule d'ouverture utilisée dans la moitié des cas est simplement «Bonjour », sans terme d'adresse (comme «Madame»), ce qui donne un ton légèrement informel, voire désinvolte. Or, ces salutations peuvent paraître inappropriées aux yeux des enseignants (Biesenbach-Lucas, 2007). Le courriel a en outre hérité des CMO les abréviations et les acronymes, ce qui est symbolique du fait que l'écriture et la lecture doivent se faire de manière rapide dans le monde numérique (Volckaert-Legrier et Bernicot, 2006). Cependant, encore une fois, ces abréviations ne sont pas toujours bien reçues par les professeurs (Stephens et al., 2009), de même que les erreurs de grammaire ou d'orthographe (Biesenbach-Lucas, 2007).

Cette hybridité de l'oral et de l'écrit pose certains problèmes dans le cas des communications entre les étudiants et leurs professeurs (Bloch, 2002), communications qui d'ailleurs ne reflètent pas toujours l'inégalité de leur relation (Biesenbach-Lucas, 2007). Alors que les professeurs s'attendent généralement à recevoir des courriels formels (Biesenbach-Lucas, 2007; Bolkan et Holmgren, 2012; Stephens et al., 2009), ce n'est pas toujours le cas en raison de l'effacement des frontières entre les professeurs et les étudiants et d'une attitude plus relâchée de ceux-ci envers la forme de leur message sous l'influence de technologies comme le clavardage (Biesenbach-Lucas, 2007).

Des études ont montré que la réception de tels courriels par les enseignants peut avoir des conséquences pour l'étudiant (Bolkan et Holmgren, 2012; Stephens et al., 2009). La réception d'un courriel informel peut en effet influencer l'affect de l'enseignant envers l'étudiant, sa perception de la crédibilité et de la qualité du message (Stephens et al., 2009), sa motivation à travailler avec l'étudiant, sa perception de la compétence de celui-ci (Bolkan et Holmgren, 2012) et celle de son succès académique futur (Bolkan et Holmgren, 2012; Stephens et al., 2009). Un tel courriel peut influencer aussi la décision de l'enseignant 
à répondre de manière positive ou négative à la demande d'aide (Bolkan et Holmgren, 2012; Stephens et al., 2009), ce qui peut avoir un impact sur la persévérance et sur le succès académique de l'étudiant.

\section{Conclusion et pistes de recherche}

La rédaction de demandes d'aide autorégulées envoyées par courriel dans un contexte d'apprentissage en classe et en ligne fait l'objet de nombreux enjeux. Ainsi, savoir formuler une demande d'aide autorégulée permet de recevoir l'aide nécessaire à la résolution du problème rencontré. De plus, savoir rédiger des courriels formels et grammaticalement corrects favorise également de bonnes relations entre l'enseignant et l'étudiant, ce qui peut influencer en retour l'attitude du premier à l'égard du second.

Voilà pourquoi nous comptons mener une étude exploratoire en nous appuyant sur le modèle de Nelson-Le Gall (1981, 1985) que Puustinen (2013) a enrichi en ajoutant des sous-composantes à chaque étape du processus et des indicateurs d'autorégulation. Notre recension des écrits nous amène cependant à différentes pistes de réflexion.

Ainsi, le modèle de Puustinen (2013) est-il transposable dans d'autres contextes de recherche? Il existe en effet des différences, au sujet des populations visées et des méthodologies de recherche, entre les recherches de Puustinen et celle que nous allons mener. Puustinen a expérimenté son modèle auprès d'enfants et d'adolescents alors que nous comptons l'utiliser auprès d'étudiants universitaires de premier cycle. Rappelons que les stratégies d'autorégulation se développent avec l'âge; certains types de demandes d'aide - comme les demandes substitutives, par exemple - pourraient être moins nombreuses, voire absentes. De plus, Puustinen a mené principalement ses recherches en présentiel, soit à l'oral et en synchrone, alors que nous étudierons les demandes d'aide dans un cours en ligne, soit à l'écrit et en mode asynchrone. Ensuite, sa collecte de données s'est faite dans un contexte expérimental et non naturel puisque les élèves étaient en contact avec un assistant de recherche et étaient filmés. Enfin, les élèves n’étaient pas notés sur les activités à réaliser alors que les étudiants de notre étude le seront : les demandes d'aide pourraient également varier en conséquence.

Dans un autre ordre d'idées, nous estimons qu'il serait utile de développer une typologie des courriels envoyés dans un cours en ligne. Nous avons vu plus haut qu'ils portent sur des thèmes très divers. Établir cette typologie pourrait nous aider à mieux orienter les étudiants vers les ressources humaines ou matérielles appropriées.

Enfin, nous examinerons les caractéristiques langagières des courriels des étudiants. Et, à partir des communications analysées, nous tenterons de dégager un profil de l'étudiant universitaire autorégulé dans un cours en ligne. 


\section{Références}

Aleven, V., Stahl, E., Schworm, S., Fischer, F. et Wallace, R. (2003). Help seeking and help design in interactive learning environments. Review of Educational Research, 73(3), 277-320. http://dx.doi.org/10.3102/00346543073003277

Ames, R. (1983). Help-seeking and achievement orientation: Perspectives from attribution theory. Dans B. DePaulo, A. Nadler et J. Fisher (dir.), New directions in helping (vol. 2, p. 165-186). New York, NY : Academic Press.

Arbreton, A. (1998). Student goal orientation and help-seeking strategy use. Dans S. A. Karabenick (dir.), Strategic help seeking: Implications for learning and teaching (p. 95-116). Mahwah, NJ : Lawrence Erlbaum Publishers.

Bandura, A. (1986). Social foundations of thought and action: A social cognitive theory. Englewood Cliffs, NJ : Prentice-Hall.

Béziat, J. (2003). Le courriel pour un tutorat de proximité en formation à distance. ÉpiNet, (57). Repéré à http://www.epi.asso.fr/revue/articles/a0306b.htm

Biesenbach-Lucas, S. (2007). Students writing emails to faculty: An examination of e-politeness among native and nonnative speakers of English. Language Learning E Technology, 11(2), 59-81. Repéré à http://lit.msu.edu/vol11num2/pdf/biesenbachlucas.pdf

Bloch, J. (2002). Student/teacher interaction via email: the social context of Internet discourse. Journal of Second Language Writing, 11(2), 117-134. http://dx.doi.org/10.1016/s1060-3743(02)00064-4

Bolkan, S. et Holmgren, J. L. (2012). “You are such a great teacher and I hate to bother you but...”: Instructors' perceptions of students and their use of email messages with varying politeness strategies. Communication Education, 61(3), 253270. http://dx.doi.org/10.1080/03634523.2012.667135

Butler, R. (2006). An achievement goal perspective on student help seeking and teacher help giving in the classroom: Theory, research, and education implications. Dans S. A. Karabenick et R. S. Newman (dir.), Help seeking in academic settings: goals, groups, and contexts (p. 15-44). Mahwah, NJ : Lawrence Erlbaum Publishers.

Cheng, K.-H., Liang, J.-C. et Tsai, C.-C. (2013). University students' online academic help seeking: The role of selfregulation and information commitments. The Internet and Higher Education, 16, 70-77. http://dx.doi.org/10.1016/j.iheduc.2012.02.002

Cosnefroy, L. (2010). Se mettre au travail et y rester : les tourments de l'autorégulation. Revue française de pédagogie, (170), 5-15. http://dx.doi.org/10.4000/rfp.1388

Cosnefroy, L. (2012). Autonomie et formation à distance. Recherche et formation, (69), 111-118. http://dx.doi.org/10.4000/rechercheformation.1752

Deasy, C., Coughlan, B., Pironom, J., Jourdan, D. et Mannix-McNamara, P. (2016). Psychological distress and help seeking amongst higher education students: findings from a mixed method study of undergraduate nursing/midwifery and teacher education students in Ireland. Irish Educational Studies, 35(2), 175-194.

http://dx.doi.org/10.1080/03323315.2016.1146157

Dunn, K. E., Rakes, G. C. et Rakes, T. A. (2014). Influence of academic self-regulation, critical thinking, and age on online graduate students' academic helpseeking. Distance Education, 35(1), 75-89. http://dx.doi.org/10.1080/01587919.2014.891426

Dweck, C. S. et Elliott, E. S. (1983). Achievement motivation. Dans P. Mussen et E. M. Heatherington (dir.), Handbook of child psychology (Vol. 4, p. 643-692). New York, NY : Wiley.

Godbole-Chaudhuri, P., Winters, F. I., Azevedo, R. et Hofman, N. (2006). Help-seeking behavior and learning with hypermedia. Dans S. Barab, K. Hay et D. Hickey (dir.), ICLS '06 Proceedings of the $7^{\text {th }}$ International Conference on Learning Sciences (p. 928-929). Bloomington, IN : International Society of the Learning Sciences.

Gough, D., Oliver, S. et Thomas, J. (2013). Learning from research: Systematic reviews for informing policy decisions: A quick guide. Repéré à https://www.alliance4usefulevidence.org/assets/Alliance-FUE-reviews-booklet-3.pdf

Hassini, E. (2006). Student-instructor communication: The role of email. Computers E Education, 47(1), 29-40. http://dx.doi.org/10.1016/j.compedu.2004.08.014 
Jézégou, A. (2010). Se former à distance : regard sur les stratégies d'autorégulation environnementale d'étudiants adultes. Savoirs, (24), 79-99. http://dx.doi.org/10.3917/savo.024.0079

Karabenick, S. (1998). Strategic help-seeking: Implications for learning and teaching. Mahwah, NJ : Erlbaum.

Karabenick, S. A. et Knapp, J. R. (1988a). Effects of computer privacy on help-seeking. Journal of Applied Social Psychology, 18(6), 461-472. http://dx.doi.org/10.1111/j.1559-1816.1988.tb00029.x

Karabenick, S. A. et Knapp, J. R. (1988b). Help seeking and the need for academic assistance. Journal of Educational Psychology, 80(3), 406-408. http://dx.doi.org/10.1037//0022-0663.80.3.406

Karabenick, S. A. et Newman, R. S. (dir.). (2006). Help seeking in academic settings: goals, groups, and contexts. Mahwah, NJ : Lawrence Erlbaum Publishers. http://dx.doi.org/10.4324/9780203726563

Kerbrat-Orecchioni, C. (2007). La « cyberpolitesse » : formes de l'adresse, ouverture et clôture dans les courriers électroniques. Quaderns de Filologia. Estudis Lingüistics, XII, 35-56. Repéré à https://ojs.uv.es/index.php/qfilologia/article/view/4089

Kitsantas, A. et Chow, A. (2007). College students' perceived threat and preference for seeking help in traditional, distributed, and distance learning environments. Computers \& Education, 48, 383-395. http://dx.doi.org/10.1016/j.compedu.2005.01.008

Kitsantas, A. et Dabbagh, N. (2009). Help seeking. Dans A. Kitsantas et N. Dabbagh (dir.), Learning to learn with integrative learning technologies (ILT): a practical guide for academic success (p. 130-142). Charlotte, NC: Information Age Pub.

Kitsantas, A., Dabbagh, N. et Dass, S. (2013). Using learning technologies to support help seeking in higher education contexts. Dans S. A. Karabenick et M. Puustinen (dir.), Advances in help-seeking research and applications: The role of emerging technologies (p. 73-97). Charlotte, NC : Information Age Pub., Inc.

Koc, S. et Liu, X. (2016). An investigation of graduate students' help-seeking experiences, preferences and attitudes in online learning. The Turkish Online Journal of Educational Technology, 15(3), 27-38. Repéré à https://files.eric.ed.gov/fulltext/EJ1106358.pdf

Makara, K. A. et Karabenick, S. A. (2013). Characterizing sources of academic help in the age of expanding educational technology: A new conceptual framework. Dans S. A. Karabenick et M. Puustinen (dir.), Advances in help-seeking research and applications: The role of emerging technologies (p. 37-72). Charlotte, NC : Information Age Pub., Inc.

Marchand, G. et Skinner, E. A. (2007). Motivational dynamics of children's academic help-seeking and concealment. Journal of Educational Psychology, 99(1), 65-82. http://dx.doi.org/10.1037/0022-0663.99.1.65

Nelson-Le Gall, S. (1981). Help-seeking: An understudied problem-solving skill in children. Developmental Review, 1(3), 224-246. http://dx.doi.org/10.1016/0273-2297(81)90019-8

Nelson-Le Gall, S. (1985). Chapter 2: Help-seeking behavior in learning. Review of Research in Education, 12(1), 55-90. http://dx.doi.org/10.3102/0091732x012001055

Nelson-Le Gall, S. et Glor-Scheib, S. (1985). Help seeking in elementary classrooms: An observational study. Contemporary Educational Psychology, 10(1), 58-71. http://dx.doi.org/10.1016/0361-476x(85)90006-2

Nicholls, J. G. (1984). Achievement motivation: Conceptions of ability, subjective experience, task choice, and performance. Psychological Review, 91(3), 328-346. http://dx.doi.org/10.1037//0033-295x.91.3.328

Park, J.-H. et Choi, H. J. (2009). Factors influencing adult learners' decision to drop out or persist in online learning. Educational Technology E' Society, 12(4), 207-217. Repéré à http://www.ifets.info/journals/12 4/18.pdf

Pintrich, P. R. (1995). Understanding self-regulated learning. New Directions for Teaching and Learning, (63), 3-12. http://dx.doi.org/10.1002/tl.37219956304

Pintrich, P. R. (2000). The role of goal orientation in self-regulated learning. Dans M. Boekaerts, P. R. Pintrich et M. Zeidner (dir.), Handbook of self-regulation (p. 451-502). San Diego, CA : Academic Press. http://dx.doi.org/10.1016/b978-012109890-2/50043-3 
Pintrich, P. R. et Schunk, D. (1996). The role of expectancy and self-efficacy beliefs. Dans P. R. Pintrich (dir.), Motivation in education: theory, research, and applications (p. 1-16). Englewood Cliffs, NJ : Merrill.

Pintrich, P. R., Smith, D. A. F., Garcia, T. et McKeachie, W. J. (1993). Reliability and predictive validity of the Motivated strategies for learning questionnaire (MSLQ). Educational and Psychological Measurement, 53(3), 801-813. http://dx.doi.org/10.1177/0013164493053003024

Poellhuber, B. (2007). Les effets de l'encadrement et de la collaboration sur la motivation et la persévérance dans les formations ouvertes et à distance soutenues par les TIC (Thèse de doctorat, Université de Montréal). Repéré à http://www2.crifpe.ca/gif/these/TheseBrunoPoellhuberFinale2007.pdf

Puustinen, M. (2012). Aider et être aidé : l'importance de la notion d'aide dans les dispositifs d'apprentissage en ligne. Revue internationale des technologies en pédagogie universitaire, 9(3), 6-9. http://dx.doi.org/10.7202/1012886ar

Puustinen, M. (2013). La demande d'aide chez l'élève : avancées conceptuelles, méthodologiques et nouvelles données. Paris : L'Harmattan.

Puustinen, M. et Bernicot, J. (2013). Seeking and obtaining online homework help: The contribution of the analysis of natural data. Dans S. A. Karabenick et M. Puustinen (dir.), Advances in help-seeking research and applications: The role of emerging technologies (p. 147-177). Charlotte, NC : Information Age Publishing.

Puustinen, M., Bernicot, J. et Bert-Erboul, A. (2011). Written computer-mediated requests for help by French-speaking students: An analysis of their forms and functions. Learning and Instruction, 21(2), 281-289. http://dx.doi.org/10.1016/j.learninstruc.2010.07.005

Puustinen, M., Bernicot, J., Volckaert-Legrier, O. et Baker, M. (2015). Naturally occurring help-seeking exchanges on a homework help forum. Computers E Education, 81, 89-101. http://dx.doi.org/10.1016/j.compedu.2014.09.010

Puustinen, M. et Rouet, J.-F. (2009). Learning with new technologies: Help seeking and information searching revisited. Computers Eं Education, 53(4), 1014-1019. http://dx.doi.org/10.1016/j.compedu.2008.07.002

Ragan, T. J. et White, P. R. (2001). What we have here is a failure to communicate: The criticality of writing in online instruction. Computers and Composition, 18(4), 399-409. http://dx.doi.org/10.1016/s8755-4615(01)00071-8

Reeves, P. M. et Sperling, R. A. (2015). A comparison of technologically mediated and face-to-face help-seeking sources. British Journal of Educational Psychology, 85(4), 570-584. http://dx.doi.org/10.1111/bjep.12088

Reffay, C. et Lancieri, L. (2006). Quand l'analyse quantitative fait parler les forums de discussion. Sciences et technologies de l'information et de la communication pour l'éducation et la formation (STICEF), 13, 1-24. Repéré à https://hal.archives-ouvertes.fr/hal-00696353

Roll, I., Aleven, V., McLaren, B. M. et Koedinger, K. R. (2011). Improving students' help-seeking skills using metacognitive feedback in an intelligent tutoring system. Learning and Instruction, 21(2), 267-280. http://dx.doi.org/10.1016/j.learninstruc.2010.07.004

Roll, I., Baker, R. S. J. d., Aleven, V. et Koedinger, K. R. (2014). On the benefits of seeking (and avoiding) help in online problem-solving environments. Journal of the Learning Sciences, 23(4), 537-560. http://dx.doi.org/10.1080/10508406.2014.883977

Roussel, P., Elliot, A. J. et Feltman, R. (2011). The influence of achievement goals and social goals on help-seeking from peers in an academic context. Learning and Instruction, 21(3), 394-402. http://dx.doi.org/10.1016/j.learninstruc.2010.05.003

Ryan, A. M., Gheen, M. H. et Midgley, C. (1998). Why do some students avoid asking for help? An examination of the interplay among students' academic efficacy, teachers' social-emotional role, and the classroom goal structure. Journal of Educational Psychology, 90(3), 528-535. http://dx.doi.org/10.1037/0022-0663.90.3.528

Ryan, A. M. et Pintrich, P. R. (1997). “Should I ask for help?” The role of motivation and attitudes in adolescents' help seeking in math class. Journal of Educational Psychology, 89(2), 329-341. http://dx.doi.org/10.1037//0022-0663.89.2.329

Ryan, A. M., Pintrich, P. R. et Midgley, C. (2001). Avoiding seeking help in the classroom: who and why? Educational Psychology Review, 13(2), 93-114. http://dx.doi.org/10.1023/A:1009013420053 
Sasseville, B. et Morel, M. (2008). L'encadrement en formation à distance : le point de vue de l'apprenant. Distances et savoirs, 6(4), 519-546. http://dx.doi.org/10.3166/ds.6.519-546

Saucier, R. (2013). Portrait des inscriptions en formation à distance (secondaire, collégial et universitaire) au Québec depuis 19951996. Montréal : Comité de liaisons interordres en formation à distance (CLIFAD).

Stephens, K. K., Houser, M. L. et Cowan, R. L. (2009). R U able to meat me: The impact of students' overly casual email messages to instructors. Communication Education, 58(3), 303-326. http://dx.doi.org/10.1080/03634520802582598

Tardif, J. (1997). Pour un enseignement stratégique : l’apport de la psychologie cognitive. Montréal, QC : Éditions Logiques.

Thouraya, D. (2007). Guider les étudiants universitaires vers l'autorégulation dans leur apprentissage en ligne. Revue internationale des technologies en pédagogie universitaire, 4(1), 20-31. http://dx.doi.org/10.18162/ritpu.2007.127

Volckaert-Legrier, O. et Bernicot, J. (2006). Le courrier électronique : un nouveau registre de la langue française? Psychologie de l'interaction, 21-22, 115-141.

Volckaert-Legrier, O., Bert-Erboul, A. et Bernicot, J. (2006). Raconter par courrier électronique : une étude de l'usage de l'orthographe chez les adolescents. Le Langage et l'Homme, 41(2), 141-156.

Weiner, B. (1979). A theory of motivation for some classroom experiences. Journal of Educational Psychology, 71(1), 3-25. http://dx.doi.org/10.1037//0022-0663.71.1.3

White, B. et Le Cornu, R. (2002). Email reducing stress for student teachers. Education and Information Technologies, 7(4), 351-357. http://dx.doi.org/10.1023/A:1020917706222

Williams, J. D. et Takaku, S. (2011). Help seeking, self-efficacy, and writing performance among college students. Journal of Writing Research, 3(1), 1-18. Repéré à http://www.jowr.org/articles/vol3 1/JoWR 2011 vol3 nr1 Williams Takaku.pdf

Woodfield, H. et Economidou-Kogetsidis, M. (2010). 'I just need more time': A study of native and non-native students' requests to faculty for an extension. Multilingua, 29(1), 77-118. http://dx.doi.org/10.1515/mult.2010.004

Zimmerman, B. J. et Schunk, D. H. (dir.). (2001). Self-regulated learning and academic achievement: Theoretical perspectives

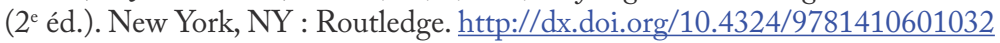

\section{Pour citer cet article}

Fatoux, C., Mottet, M. et Rouissi, S. (2018). État des connaissances sur la demande d'aide : quel apport pour la formation universitaire en ligne? Formation et profession, 26(1), 43-54. http://dx.doi.org/10.18162/fp.2018.373 